\title{
PREVENTION \& CONTROL STRATEGIES FOR NOSOCOMIAL INFECTION
}

\author{
AUTHORS: \\ SURIYA NARAYANAN HARIKRISHNAN (a), DATTATREYA MUKHERHEE (a) \\ SUFIA IMAM (a), AAYUSHI RAJ SINHA (b) \\ MBBS \\ a) JINAN UNIVERSITY, P.R CHINA \\ b) GEMEDI TEACHING UNIVERSITY
}




\section{INTRODUCTION}

Nosocomial infection is every infectious process appearing during hospital stays, despite its clinical picture carrier status and time of manifestation - during hospital treatment or after discharge.

A nosocomial infection is contracted because of an infection or toxin that exists in a certain location, such as a hospital. People now use nosocomial infections interchangeably with the terms health-care associated infections (HAIs) and hospital-acquired infections. For a HAI, the infection must not be present before someone has been under medical care.

The responsibility of HAI prevention is with the healthcare facility. Hospitals and healthcare staff should follow the recommended guidelines for sterilization and disinfection. However, due to the nature of healthcare facilities, it's impossible to eliminate 100 percent of nosocomial infections.

Prevention of nosocomial infections requires an integrated, monitored, programme which includes the following key components:

(1.) limiting transmission of organisms between patients in direct patient care through adequate handwashing and glove use, and appropriate aseptic practice, isolation strategies, sterilization and disinfection practices, and laundry

(2.) controlling environmental risks for infection

(3.) protecting patients with appropriate use of prophylactic antimicrobials, nutrition, and vaccinations

(4.) limiting the risk of endogenous infections by minimizing invasive procedure, and promoting optimal antimicrobial use

(5.) surveillance of infections, identifying and controlling outbreaks

(6.) prevention of infection in staff members

(7.) enhancing staff patient care practices, and continuing staff education.

Infection control is the responsibility of all health care professionals - doctors, nurses, therapists, pharmacists, engineers and others.

\section{PREVENTION OF NOSOCOMIAL INFECTIONS}

The two primary routes of exposure to infectious diseases that are of concern for HCWs are related to fomites (including, in this discussion, direct contact with people) and airborne transmission although cuts/injection (e.g., needle sticks) is also commonly mentioned. Both of these routes can be initiated by a carrier. The most effective method for eliminating microbes is sterilization. This generally involves the use of moist heat, that is, 15 pounds of pressure per square inch (psi) for 15 minutes at $121^{\circ} \mathrm{C}$. Although these practices are of great importance, one of the most critical is hand washing. 
Since most NIs in hospitals are associated with the urinary tract, catheters should be employed only when necessary. Catheters should not be employed longer than required and should be removed as soon as possible (WHO 2002). Practices of this nature can reduce the rate of these infections by $\mathbf{4 0 \%}$ (Curtis 2008). Hand washing is also important in these infections and may be the cause of up to $15 \%$ of NI events.

However, even vigorous cleaning twice a day has not been shown to be effective in reducing the rates of UTIs. In general, one of the identified problems is that hospital cleaning personnel receive little or no training on good cleaning practices. The most effective method, overall, for preventing NIs is effective hand washing. However, even with effective hand washing, more effective cleaning practices are needed.

Some general measures for infection control include:

- Screening the ICU to see if people with HIAs need to be isolated.

- Identifying the type of isolation needed, which can help to protect others or reduce chances of further infection.

- Observing hand hygiene, which involves washing hands before and after touching people in the hospital.

- Wearing appropriate gear, including gloves, gowns, and face protection.

- Cleaning surfaces properly, with recommended frequency.

- Making sure rooms are well ventilated.

To reduce the risk of UTIs, your healthcare provider can:

- Follow the aseptic insertion technique to minimize infection.

- Insert catheters only when needed and remove when no longer needed.

- Change catheters or bags only when medically indicated.

- Make sure the urinary catheter is secured above the thigh and hanging below the bladder for unobstructed urine flow.

- Keep a closed drainage system. 


\section{Isolation of Infection:}

Isolation of infection is defined as the use of all measures that prevent the direct and indirect transmission of an infectious agent from the source or the reservoir to a recipient host.

There are two main functions of isolation, according to the relative hazard of the transmission of the infectious agent:

$\checkmark$ Protective isolation

$\checkmark$ Infective isolation

Protective isolation is used for immuno-compromised patients in order to protect them from the transmission of an infectious agent from an immuno-competent person or other source to prevent the serious consequences of acquiring an infection. This type of isolation is used, for example, in bone marrow or kidney transplantation, when immuno-suppression is used to decrease the function of the immune system in recipients to avoid organ rejection. It is also used for newborn babies with congenital immune disorders.

Infective isolation is used when the host, regardless of the immune status, harbors a hazardous agent that is likely to transmit an infectious agent into the environment. Here the aim is to prevent the transmission to other possible hosts in the environment.

\section{Isolation at the source of infection}

Decontamination aims to remove or to kill the infectious agents in the source and/or within the mode of transmission.

The effect of decontamination depends on the method used, on the initial amount of the micro-organism, and on the resistance of the micro-organism. This is expressed as the reduction of the micro-organism under the defined conditions of the decontaminating method, per unit of time.

The choice of decontamination method depends on the level of the risk of clinical infection.

$>$ high risk - critical instruments that penetrate sterile tissues or blood vessels, or have contact with mucosa (surgical items, endoscopes, syringes, needles, catheters, etc.) even if contaminated initially with microbes with low virulence. These items should be free of any kind of viable micro-organisms.

$>$ intermediate risk - semi-critical items that have direct contact with intact skin (bedclothes, stethoscope, blood pressure cuffs). Here, pathogenic microbes causing 
infectious diseases of the skin, or able to transmit other potential pathogens, should be absent.

low risk - non-critical items that do not have direct contact with people, and especially with patients (e.g. walls, floor). But if Bacillus anthracis - a very dangerous infectious disease $\sim$ is found on the walls or floor the environment cannot then be described as "low risk". Low risk items should be free of absolute pathogen.

\section{Disinfection:}

Disinfection is defined as the killing of microbes in both the animate and inanimate environment. The resistance of micro-organisms is not uniform against different disinfectant agents. Complete disinfection (= sterilization) means that the disinfectant (sterilant) kills even the most resistant infectious agent on the resistance scale. Disinfection is said to be incomplete if the disinfectant does not kill every type of micro-organism (selective killing).

Two main types of disinfection are used:

* physical

* chemical.

\section{Complete Disinfection (sterilization)}

Sterilization, or complete disinfection, is the process whereby items become sterile by killing all the living organisms. Sterilization in medicine began with the improvement of surgical asepsis in order to develop sterilization methods that kill any potential infectious agent occurring on earth, without damaging the sterilized items.

The uses of sterilization are:

- decontamination of high-risk items used for procedures on the human body, preventing procedure-associated infections if there is a high risk of developing infectious disease.

- within the pharmaceutical industry

- decontamination of infectious waste

- other non-medical uses (preservation of food, etc.)

\section{Disinfection of the inanimate environment:}

As the inanimate environment can serve as the source or the vehicle of the transmission of an infectious agent, it is necessary to decontaminate it in order to control the infection. In health care settings the next parts of the 
inanimate environment that needs decontamination are:

- surfaces

- textiles

- equipment

- water

- air.

$\underline{\text { Transmission from environment }}$

Unhygienic environment serves as the best source for the pathogenic organism to prevail. Air, water and food can get contaminated and transmitted to the patients under healthcare delivery. There must be policies to ensure the cleaning and use of cleaning agents on walls, floor, windows, beds, baths, toilets and other medical devices. Proper ventilated and fresh filtered air can eliminate airborne bacterial contamination. Regular check of filters and ventilation systems of general wards, operating theatres and ICUs must be maintained and documented.

$\underline{\text { Transmission from staff }}$

Infections can be transferred from healthcare staff. It is the duty of healthcare professionals to take role in infection control. Personal hygiene is necessary for everyone so staff should maintain it. Hand decontamination is required with proper hand disinfectants after being in contact with infected patients. Safe injection practices and sterilized equipment should be used. Use of masks, gloves, headcovers or a proper uniform is essential for healthcare delivery.

\section{Hospital waste management}

Waste from hospitals can act as a potential reservoir for pathogens that needs proper handling. 10-25\% of the waste generated by healthcare facility is termed as hazardous. Infectious healthcare waste should be stored in the area with restricted approach. Waste containing high content of heavy metals and waste from surgeries, infected individuals, contaminated with blood and sputum and that of diagnostic laboratories must be disposed off separately. Healthcare staff and cleaners should be informed about hazards of the waste and it's proper management.

Nosocomial infections are associated with a great deal of morbidity, mortality and increased financial burden. Intensive care is a risk factor for the emergence of antibiotic resistant bacteria. Gram-positive bacteria have overtaken Gram-negative organisms as the 
predominant cause of nosocomial infections. Inadequate antibiotic therapy is associated with poor outcome and particularly with bacterial resistance. Infection control measures are important for the effective control, prevention and treatment of infection. Knowledge of emerging pathogens and resistance profile is essential for treatment against nosocomial infections. Shorter duration of treatment and correct dosage of antibiotic therapy is recommended to reduce the selection pressure for resistant isolates. Hand washing is the single most important measure to prevent nosocomial infections. Gloves must not be used as a substitute for hand washing; they must be washed on glove removal.

Protective garments are necessary for health providers exposed to body fluids, for example sweat, oropharyngeal fluids, blood or urine. Gloves and aprons should be worn for handling body fluids. High efficiency particulate air (HEPA) filter masks are recommended for sputum smear positive patients with tuberculosis, particularly for cough-inducing procedures. Hands must be washed after glove removal as contamination of the hands can still occur.

The use of invasive procedures increases the risk of nosocomial infections. For venous access, this risk can be reduced by use of specific sites such as subclavian vein rather than internal jugular or femoral veins. Tunneling the catheter reduces the risk of nosocomial infection. Antimicrobial impregnated catheters can reduce catheter related infections. The use of a strict, aseptic technique is paramount in the insertion of intravascular catheters.

\section{CONTROL OF NOSOCOMIAL INFECTION}

Despite of significant efforts made to prevent nosocomial infections, there is more work required to control these infections. In a day, one out of 25 hospital patients can acquire at least a single type of nosocomial infection.

\section{$\underline{\text { Infection control programs }}$}

Healthcare Institutes should devise control programs against these infections. Administration, workers and individuals admitted or visiting hospital must take into account such programs to play their role in prevention of infections. 


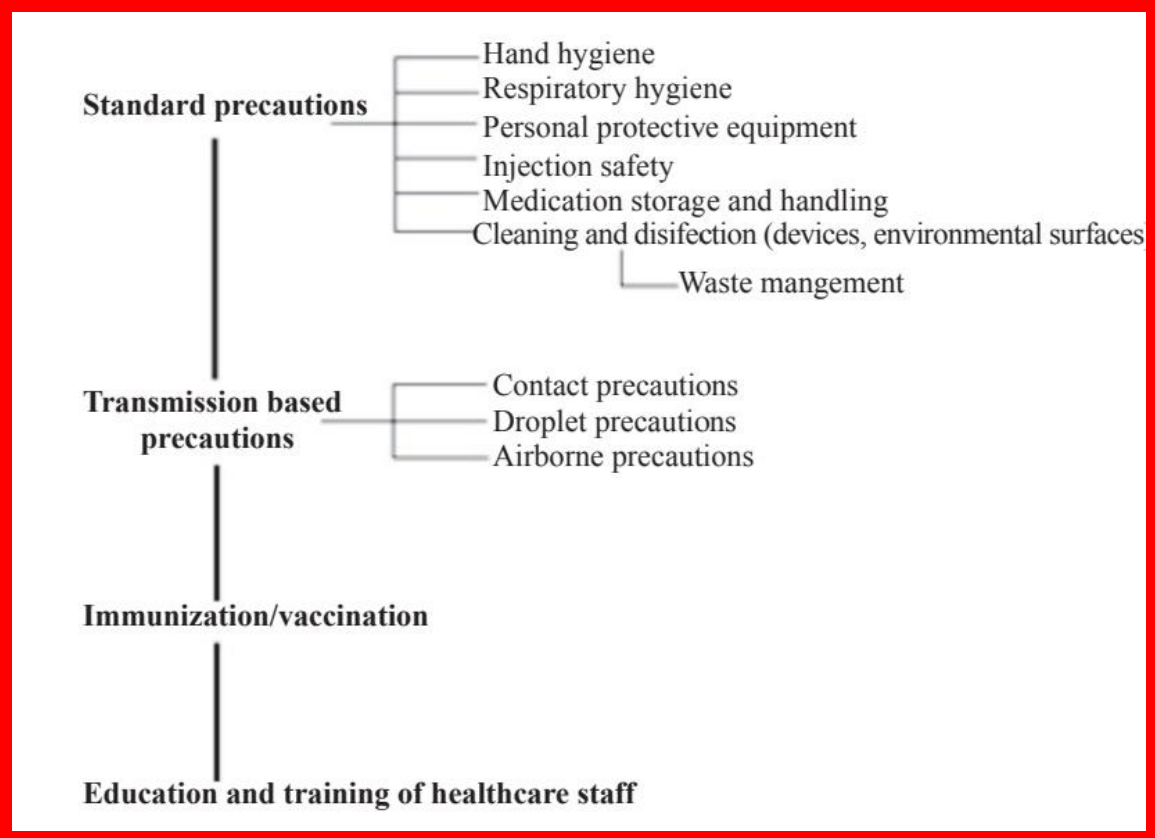

Nosocomial infections can be controlled by practicing infection control programs, keep check on antimicrobial use and its resistance, adopting antibiotic control policy. Efficient surveillance system can play its part at national and international level. Efforts are required by all stakeholders to prevent and control nosocomial infections.

\section{CONCLUSION:}

NIs are becoming of greater concern in the HCI and general community. It appears that the rate of NI will continue to increase in the future and will further spread in the community environment. For the HCI, the costs associated with these infections are now becoming critical, and NIs are a major issue in cost containment. With increased burden of nosocomial infections and antimicrobial resistance, it has become difficult for healthcare administrations and infection control committees to reach the goal for elimination of intervals. However, by practicing sound and healthy ways for care delivery designed by infection control committees, controlling transmission of these infections using appropriate methods for antimicrobial use, the resistance in emerging pathogens against antimicrobials can be reduced easily. An efficient surveillance method guided by WHO can help healthcare institutes to devise infection control programs. Proper training of hospital staff for biosafety, proper waste management and healthcare reforms and making general public aware of these endemic infections can also help in reduction of nosocomial infectios. 